\title{
Male- and female-derived somatic and germ cell specific toxicity of silver nanoparticles in mouse
}

Jae Woong Han $^{1 \#}$, Jae-KyoJeong ${ }^{1 \#}$, Sangiliyandi Gurunathan ${ }^{1 \#}$, Yun-Jung Choi ${ }^{\# 1}$, Joydeep Das $^{1}$, Deug-Nam Kwon ${ }^{1}$, Ssang-Goo Cho ${ }^{1}$, Chankyu Park ${ }^{1}$, Jin-Ki Park ${ }^{2 \dagger}$, and Jin-Hoi $\operatorname{Kim}^{1 \dagger}$

${ }^{1}$ Department of Animal Biotechnology, Konkuk University, Seoul 143-701, Republic of Korea.

${ }^{2}$ Animal Biotechnology Division, National Institute of Animal Science, Suwon, Korea.

\#Jae Woong Han, Jae-Kyo Jeong, Sangiliyandi Gurunathan, and Yun-Jung Choi contributed equally to this work.

To whom correspondence should be addressed ${ }^{\dagger}$ :

Jin-Hoi Kim, PhD: jhkim541@konkuk.ac.kr

Running title: silver nanoparticles toxicity in mouse germ cells 


\section{Supplementary methods}

\section{Synthesis and characterization of AgNPs}

Synthesis and characterization of AgNPs were performed according to the method described previously (Gurunathan et al., 2009). The Dynamic Light Scattering (DLS), UVvisible spectra and X-ray diffraction (XRD) of AgNPs were measured by using Zetasizer Nano ZS90 (Malvern Instruments; Limited; UK), Optizen POP (Mecasys, South Korea), and Bruker D8 Discover (Bruker AXS GmBH, Karlsruhe, Germany) instruments respectively in National Center for Inter-University Reserch Facilities (Seoul National University, South Korea). The high resolution XRD patterns were measured at $3 \mathrm{~kW}$ with $\mathrm{Cu}$ target using a scintillation counter $\left(\lambda=1.5406^{\circ} \mathrm{A}\right)$ at $40 \mathrm{kV}$ and $40 \mathrm{~mA}$; recorded range value is $2 \theta=5^{\circ}-$ $80^{\circ}$. The EDX spectra were performed to analyze the purity of the material and the complete chemical composition of synthesized silver nanoparticles. Transmission electron microscopy (TEM), using a JEM-1200EX microscope was performed to determine the size and morphology of AgNPs. TEM images of AgNPs were obtained at an accelerating voltage of $300 \mathrm{kV}$.

\section{Transmission electron microscopy (TEM) analysis}

After fixation of arterial specimens in $2.5 \%$ glutaraldehyde (Redding, CA, USA) in PBS ( $\mathrm{pH}, 7.2$ ), samples were postfixed in $1 \%$ osmium tetroxide (Heraeus, Boksburg, South Africa), dehydrated in a graded series of ethanol baths and propylene oxide (Acros Organics, New Jersy, USA), and then embedded in epoxy resin [EMbed 812 containing nadic methyl anhydride (NMA), dodenyl succinic anhydride (DDSA), and DMP-30] as described previously (Kuo, 2007). Serial ultrathin sections were cut on an LKB-III ultratome (LEICA, Wetzlar, Germany). Sections were stained with uranyl acetate and lead citrate (Redding, CA, USA). Sections were examined under a Hitachi H7600 electron microscope (Hitachi, Tokyo, 
Japan) at an accelerating voltage of $100 \mathrm{kV}$.

\section{Real-time quantitative reverse transcription polymerase chain reaction}

Polymerase chain reaction (PCR) was performed according to the instructions of the realtime PCR machine manufacturer (ViiA ${ }^{\mathrm{TM}} 7$, Applied Biosystems, Foster City, CA). For the analysis of cytokines, we determined that a $400-\mu \mathrm{L}$ aliquot of blood collected after two different doses $(0.5 \mathrm{mg} / \mathrm{kg}$ and $1.0 \mathrm{mg} / \mathrm{kg})$ of AgNPs provided sufficient mRNA for gene expression studies. We successfully quantified the expression levels of tumor necrosis factor $\alpha($ TNF- $\alpha$ ), INF- $\gamma$, interleukins-6 (IL-6) and - $\beta$ (IL- $\beta$ ), and monocyte chemoattractant protein1 (MCP-1) with this approach. The RT-PCR primer sets are shown in Supplementary Table 1. Target gene expression levels were normalized to GAPDH gene expression.

\section{Hematological analysis}

Hematological and clinical chemistry analyses were performed on blood samples obtained at autopsy. Blood was collected in EDTA-coated tubes. Hematological parameters included white blood cell (WBC), lymphocyte (LY), monocyte (MO), neutrophil (NE), eosinophil (EO), and red blood cell (RBC) counts; mean corpuscular volume (MCV); hematocrit (HCT);mean corpuscular hemoglobin $(\mathrm{MCH})$; mean corpuscular hemoglobin concentration (MCHC); hemoglobin (HGB); red blood cell distribution width (RDW); platelet (PLT) count; and mean platelet volume (MPV). All hematological parameters were determined using an Advia 120 Hematology Analyzer (Siemens, Germany). Blood smears were also prepared for visual evaluation. 


\section{Histopathology and TUNEL staining}

The kidney, liver, lung, spleen, testes, and ovaries were removed and examined microscopically for gross lesions. All specimens were fixed in $4 \%$ paraformaldehyde and embedded in paraffin. Cross-sections were obtained, stained with hematoxylin and eosin, and mounted with Vectashield medium. Cross-sections were examined under a light microscope. 
Supplementary Table 1.

\begin{tabular}{|c|c|c|}
\hline Gene & Primer & $\mathbf{T M}\left({ }^{\circ} \mathrm{C}\right)$ \\
\hline \multirow{2}{*}{ 3ß-HSD } & F: CTGAATGTTACTGGCAAATTCTC & \multirow[b]{2}{*}{58} \\
\hline & R: TGTAAAATGGACGCAGCAGGAA & \\
\hline \multirow{2}{*}{ 17ß-HSD3 } & F: GTTCTCGCAGCACCTTTTTC & \multirow[b]{2}{*}{58} \\
\hline & R: ACAATCTTCACACCGCTTCC & \\
\hline \multirow{2}{*}{ CYP19 } & F: CACCCTTCCAAGTGACAGGA & \multirow[b]{2}{*}{58} \\
\hline & R: AAAAAAGTAAAGTTCTATGGGAA & \\
\hline \multirow{2}{*}{ CYP17 } & F: TTTTGGCCCAAGTCAAAGAC & \multirow{2}{*}{53} \\
\hline & R: CCCTTCTTCACGAGCACTTC & \\
\hline \multirow{2}{*}{ GATA4 } & F: CCCTACCCAGAATACATGG & \multirow[b]{2}{*}{58} \\
\hline & R: ACATATCGAGATTGGGGTGTCT & \\
\hline \multirow{2}{*}{ STAR } & F: GCAGCAGGCAACCTGGTG & \multirow[b]{2}{*}{58} \\
\hline & R: TGATTGTCTTCGGCAGCC & \\
\hline \multirow{2}{*}{ CYP11a1 } & F: CGATACTCTTCTCATGCGAG & \multirow[b]{2}{*}{58} \\
\hline & R: CTTTCTTCCAGGCATCTGAAC & \\
\hline \multirow{2}{*}{ Gapdh } & F: AGGTCGGTGTGAACGGATTTG & \multirow{2}{*}{58} \\
\hline & R: TGTAGACCATGTAGTTGAGGTCA & \\
\hline \multirow{2}{*}{ Stra8 } & F: ATTATAATGGCCACCCCTGG & \multirow{2}{*}{58} \\
\hline & R: CCAGGCTTTCTTCCTGTTC & \\
\hline \multirow{2}{*}{ Sohlh1 } & F: TGACAACGCGCTCTGGCG & \multirow[b]{2}{*}{60} \\
\hline & R: TGCCTCAGTTTGATGGCC & \\
\hline \multirow{2}{*}{ SCP1 } & F: AGGTTGAGAAAGCCAAAGCA & \multirow[b]{2}{*}{60} \\
\hline & R: GTCTCCAAAGCAATCTTCGC & \\
\hline \multirow{2}{*}{ SCP3 } & F: CATTCTGGGAAATCTGGGAA & \\
\hline & R: GGAGCCTTTTCATCAGCAAC & 60 \\
\hline GFRA1 & F: GAACTACGCAGACTGCCTCC & \\
\hline GFRAI & R : ATCTTCCAGGTCATTGCCAC & 58 \\
\hline & F: AACGGTTCCTGGACAGTTTG & \\
\hline PLZF & R: ACTCGCACAGCTGGTAGGTT & 60 \\
\hline & F: AAGGCAAAATCATGCCAAAC & \\
\hline Dazl & R: AAAATCAAAGGACGTGGCTG & 58 \\
\hline & F: CACCAGGACACCAACATCTG & \\
\hline Mde1 & R: TCTTCCCCTGATTTCCCTCT & 58 \\
\hline & F: CTGCTGACTGAGGATGACCA & \\
\hline Dnmt3L & R: GCTTGCTCCTGCTTCTGACT & 58 \\
\hline & F: CAACAAGAATGTGGTGGTCG & \\
\hline Miwi2 & R: GTCATGCAGACTTTGAGGCA & 58 \\
\hline & F: AATTTTGGAGGTTTCCCC & \\
\hline Hrb & R: TGCTGTCTGATGACTCTGGG & 60 \\
\hline & F: GCTGCATGTTGGAGATGCTA & \\
\hline GOPC & R: ATCTCGCCACTGCTGTCTTT & 58 \\
\hline & F: AGCAAAAGCAGGAGCAGATG & \\
\hline PRM1 & R: CCTTATGGTGTATGAGCGGC & 58 \\
\hline & F: GTTCGCTACCGAATGAGGAG & \\
\hline PRM2 & R: CCATAGTCCTCTACGCGCTC & 58 \\
\hline & F: ACAGAGCAGGAAGCCCAGTA & \\
\hline FIGLA & R: ACTCGCACAGCTGGTAGGTT & 60 \\
\hline & F: ACAAACGCCATGAGATTTCC & \\
\hline NOBOX & $\mathrm{R}$ :AACAGGGCCAGGTTCTAGGT & 58 \\
\hline
\end{tabular}




\begin{tabular}{|c|c|c|}
\hline \multirow{2}{*}{ LHX8 } & F: CAGTTCGCTCAGGACAACAA & \multirow[b]{2}{*}{58} \\
\hline & R: AGCCATTTCTTCCAACATGG & \\
\hline \multirow{2}{*}{ FOXO3A } & F: GATGATGATGGACCCCTGTC & \multirow{2}{*}{58} \\
\hline & R: TCTTGGCGGTATATGGGAAG & \\
\hline \multirow[b]{2}{*}{$\mathbf{A B P}$} & F:CACGCAGAATTCAGTCTCCA & \multirow[b]{2}{*}{60} \\
\hline & R:CCTGTTCCAAGAGCAAGGAG & \\
\hline \multirow[b]{2}{*}{ GATA1 } & F:GATGGAATCCAGACGAGGAA & \multirow[b]{2}{*}{60} \\
\hline & R:CAGCAGAGGTCCAGGAAAAG & \\
\hline \multirow[b]{2}{*}{ Inhibin B } & F:ACAGGCCCTTTGTAGTGGTG & \multirow[b]{2}{*}{60} \\
\hline & R:AGCCGAAAGTCGATGAAGAA & \\
\hline \multirow[b]{2}{*}{ Serotonin receptor } & F:GGAGAAAAGGCTGCAGTACG & \multirow[b]{2}{*}{60} \\
\hline & R:ATAACCAGGCAGGACACAGG & \\
\hline \multirow[b]{2}{*}{ TNF- $\alpha$} & F:CATCTTCTCAAAATTCGAGTGACAA & \multirow[b]{2}{*}{53} \\
\hline & R: TGGGAGTAGACAAGGTACAACCC & \\
\hline \multirow[b]{2}{*}{ INF- $\gamma$} & F: TCAAGTGGCATAGATGTGGAAGAA & \multirow[b]{2}{*}{58} \\
\hline & R:TGGCTCTGCAGGATTTTCATG & \\
\hline \multirow[b]{2}{*}{ IL-1B } & F: AGGTCGCTCAGGGTCACAAG & \multirow[b]{2}{*}{58} \\
\hline & R:GTGCTGCCTAATGTCCCCTTGAATC & \\
\hline \multirow[b]{2}{*}{ IL-6 } & F: GACAACTTTGGCATTGTGG & \multirow[b]{2}{*}{58} \\
\hline & R: ATGCAGGGATGATGTTCTG & \\
\hline \multirow[b]{2}{*}{ MCP-1 } & F: AAGTTGACCCGTAAATCT & \multirow[b]{2}{*}{58} \\
\hline & R: CTAGTTCACTGTCACACT & \\
\hline
\end{tabular}




\section{Supplementary Table 2.}

\begin{tabular}{|c|c|c|c|c|c|c|}
\hline & \multicolumn{3}{|c|}{ Male } & \multicolumn{3}{|c|}{ Female } \\
\hline Parameters & PBS & $\begin{array}{c}500 u g / k g \\
\text { AgNP }\end{array}$ & $\begin{array}{c}\text { 1000ug/kg } \\
\text { AgNP }\end{array}$ & PBS & $\begin{array}{c}500 \mathrm{ug} / \mathrm{kg} \\
\text { AgNP }\end{array}$ & $\begin{array}{c}\text { 1000ug/kg } \\
\text { AgNP }\end{array}$ \\
\hline $\mathrm{WBC}(\mathrm{K} / \mu \mathrm{l})$ & $5.635( \pm 2)$ & $5.336( \pm 1.29)$ & $4.58( \pm 0.96)$ & $4.11( \pm 1.26)$ & $4.705( \pm 0.97)$ & $3.206( \pm 0.48)$ \\
\hline $\mathbf{L Y}(\%)$ & $82.69( \pm 6.92)$ & $78.97( \pm 3.36)$ & $82.46( \pm 3.53)$ & $82.315( \pm 4.45)$ & $77.5575( \pm 5.39)$ & $88.706( \pm 3.72)$ \\
\hline $\operatorname{MO}(\%)$ & $3.37( \pm 1.29)$ & $4.776( \pm 0.55)$ & $3.76( \pm 0.82)$ & $4.09( \pm 1.06)$ & $4.215( \pm 0.5)$ & $2.474( \pm 0.4)$ \\
\hline $\mathrm{NE}(\%)$ & $10.32( \pm 4.85)$ & $13.002( \pm 2.46)$ & $12.25( \pm 2.78)$ & $13.695( \pm 2.86)$ & $8.27( \pm 3.57)^{*}$ & $5.93( \pm 2.89)^{*}$ \\
\hline $\operatorname{EO}(\%)$ & $3.081( \pm 3.56)$ & $1.93( \pm 1.03)$ & $1.00( \pm 0.85)$ & $0.8875( \pm 0.3)$ & $10.1475( \pm 8)$ & $3.016( \pm 3.32)$ \\
\hline $\mathbf{B A}(\%)$ & $0.37( \pm 0.31)$ & $0.644( \pm 0.23)$ & $0.345( \pm 0.24)$ & $0.6375( \pm 0.33)$ & $0.475( \pm 0.19)$ & $0.456( \pm 0.13)$ \\
\hline $\operatorname{RBC}(M / \mu \mathrm{l})$ & $6.605( \pm 1.08)$ & $7.406( \pm 0.34)$ & $6.995( \pm 1.42)$ & $6.755( \pm 1.09)$ & $6.805( \pm 0.91)$ & $6.182( \pm 0.62)$ \\
\hline $\operatorname{MCV}(f L)$ & $58.62( \pm 3.2)$ & $57.306( \pm 1.63)$ & $61.4275( \pm 2.87)$ & $58.335( \pm 0.6)$ & $58.61( \pm 4.32)$ & $57.368( \pm 1.39)$ \\
\hline НСТ(\%) & $38.97( \pm 8.23)$ & $42.706( \pm 2.5)$ & $42.9275( \pm 8.82)^{*}$ & $45.3975( \pm 6.53)$ & $38.0625( \pm 4.48)$ & $35.412( \pm 2.94)$ \\
\hline $\operatorname{MCH}(\mathbf{p g})$ & $15.81( \pm 0.59)$ & $16.582( \pm 0.24)$ & $19.69( \pm 5.97)$ & $16.3( \pm 0.28)$ & $15.955( \pm 0.72)$ & $15.686( \pm 0.34)^{*}$ \\
\hline $\operatorname{MCHC}(\mathrm{g} / \mathrm{dL})$ & $27.03( \pm 1.47)$ & $28.116( \pm 0.4)$ & $31.96( \pm 8.9)$ & $27.9425( \pm 0.55)$ & $27.3575( \pm 1.18)$ & $27.038( \pm 0.91)$ \\
\hline $\operatorname{Hgb}(g / d L)$ & $10.48( \pm 1.96)$ & $12.116( \pm 0.39)$ & $13.1675( \pm 0.99)$ & $12.695( \pm 1.91)$ & $12.1025( \pm 1.43)$ & $9.534( \pm 1.07)$ \\
\hline $\operatorname{RDW}(\%)$ & $16.23( \pm 0.49)$ & $16.62( \pm 0.6)$ & $15.85( \pm 0.85)$ & $16.325( \pm 0.34)$ & $16.45( \pm 0.66)$ & $16.4( \pm 0.41)$ \\
\hline $\operatorname{PLT}(K / \mu \mathrm{l})$ & $763.16( \pm 228.73)$ & $827.4( \pm 343.1)$ & $1145.25( \pm 298.6)$ & $877.5( \pm 121.05)$ & $826.25( \pm 160)$ & $735( \pm 234.17)$ \\
\hline MPV(fL) & $7.37( \pm 0.88)$ & $6.404( \pm 0.57)$ & $6.6125( \pm 0.28)$ & $6.6275( \pm 0.12)$ & $6.965( \pm 0.13)$ & $7.866( \pm 0.6)$ \\
\hline
\end{tabular}




\section{Supplementary Table 1.}

Primers used for quantitative real-time polymerase chain reaction

\section{Supplementary Table 2.}

Effect of silver nanoparticles on blood parameters 
a

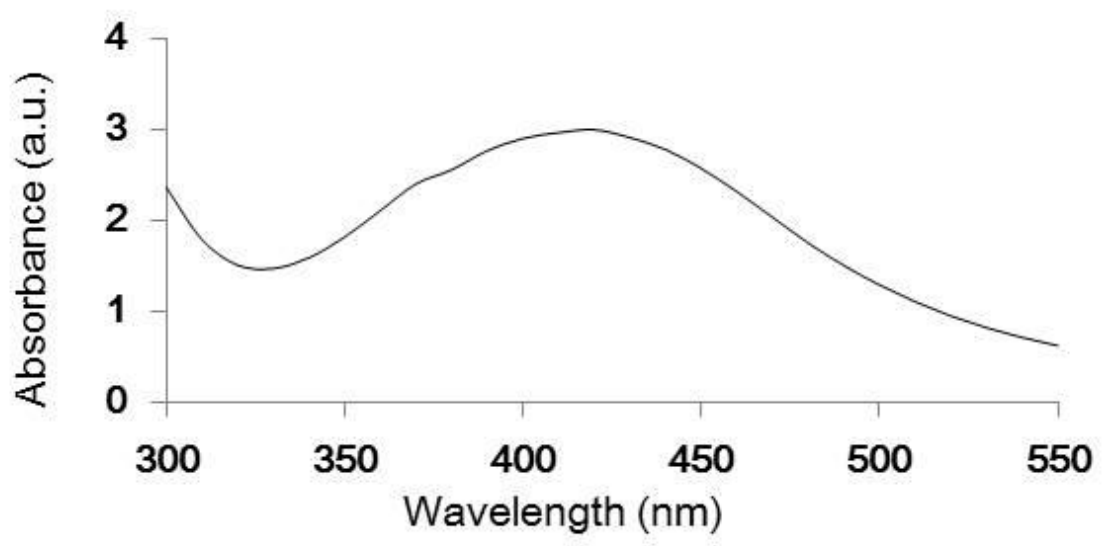

b

Size Distribution by Intensity
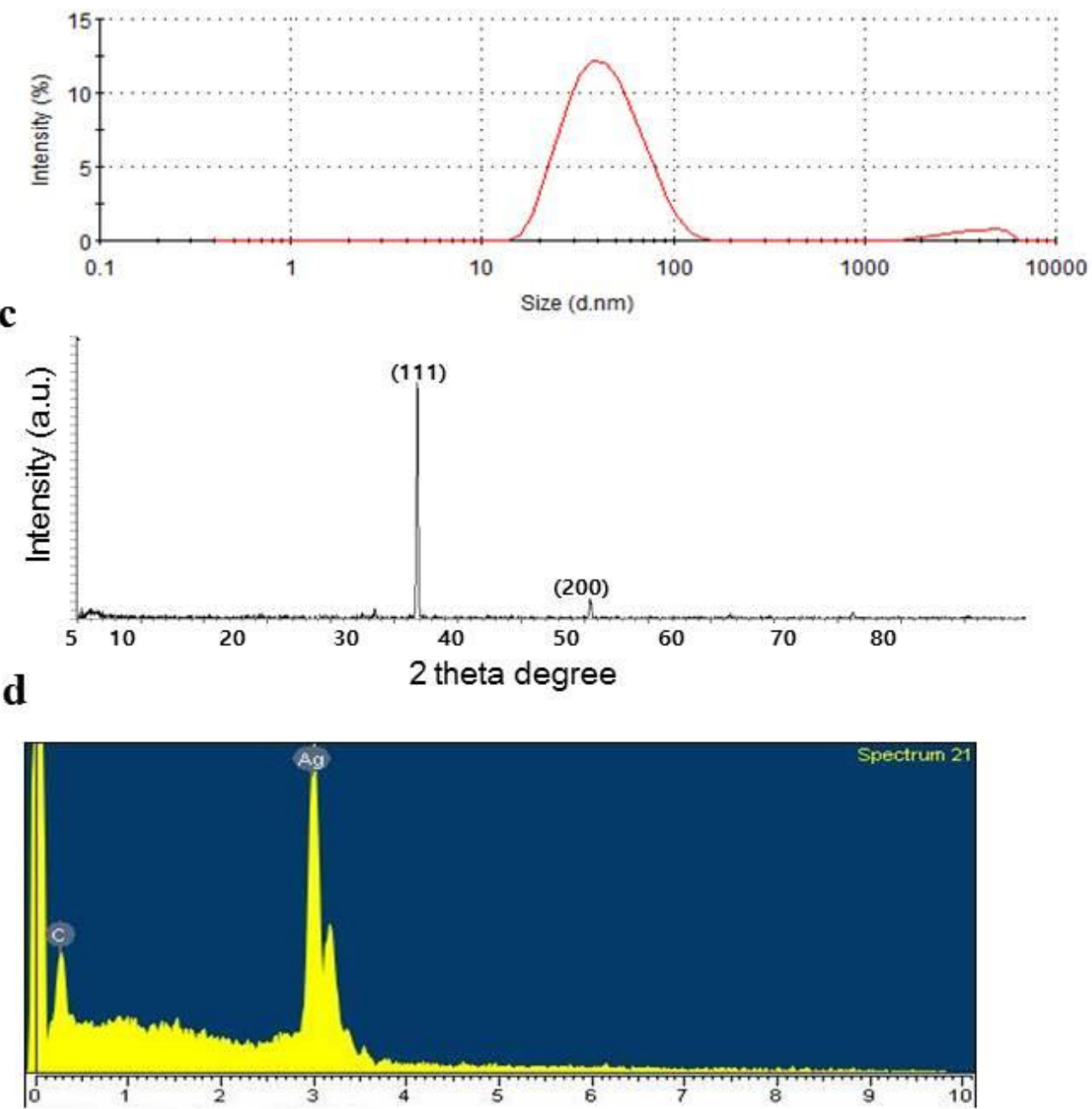

Supplementary Fig. 1. UV-visible spectrum (a), size distribution analysis by DLS (b), XRD 
(c) and EDX spectra analysis (d) of AgNPs.
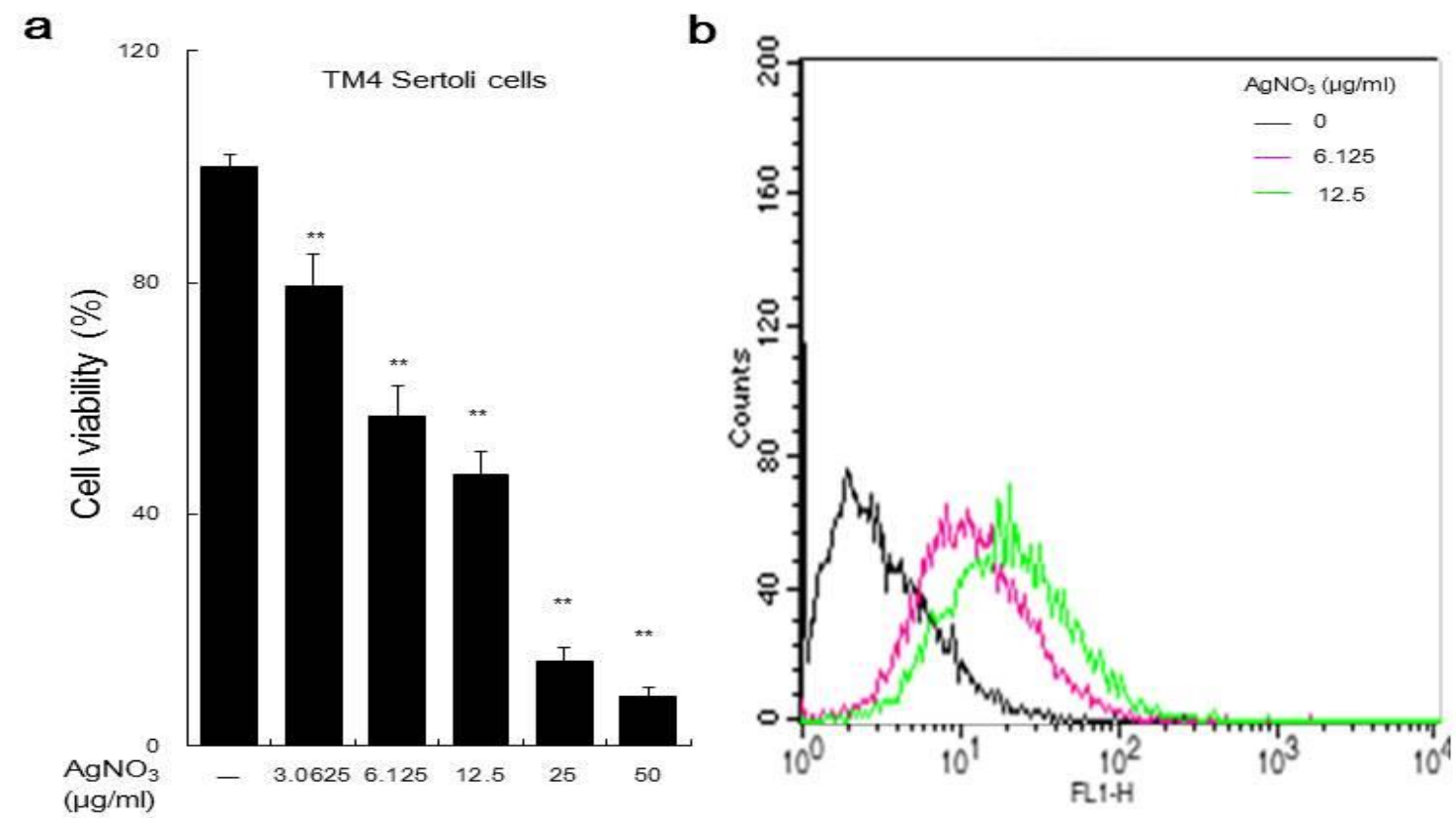

Supplementary Fig. 2.(a) TM4 Cell viability relative to the control (100\%) was measured using the Cell Counting Kit-8 (CCK-8), exposed to different concentrations of $\mathrm{AgNO}_{3}$ for 24 hr. Experiments were performed in triplicate, and values are expressed as mean \pm SEM of three independent experiments. (b) $\mathrm{H}_{2}$-DCFDA-positive cells measured by flow cytometry in TM4 cells treated with different concentrations of $\mathrm{AgNO}_{3}$ for $24 \mathrm{~h}$. M2 represents the 
population of $\mathrm{H}_{2}$-DCFDA-positive cells. $* * P<0.01$ compared with the control.

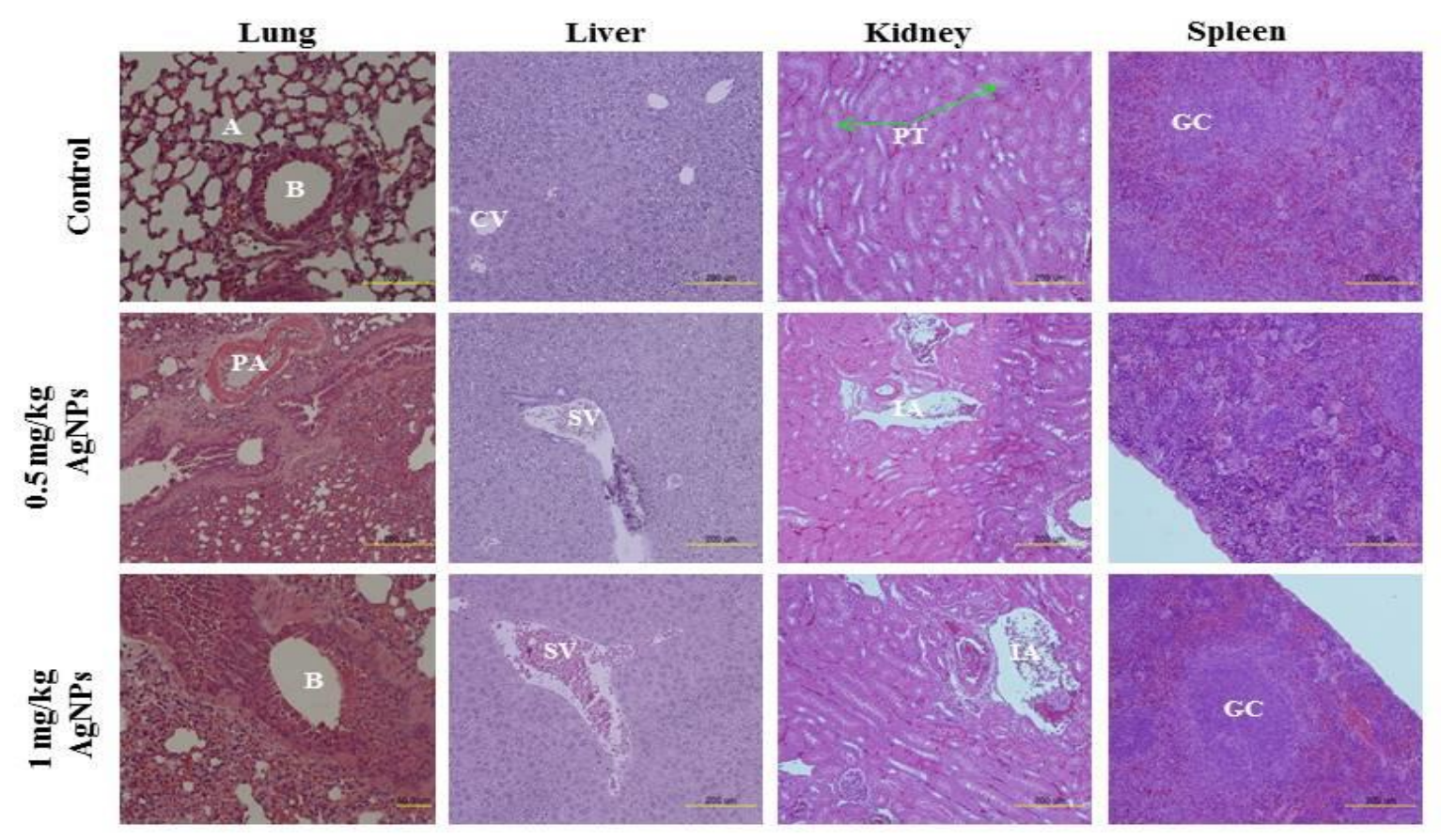

Supplementary Fig. 3. H\&E staining of lung, liver, kidney, and spleen of control and AgNPs-treated mice. A, alveoli; B, bronchiole: PA, pulmonary artery; PT, proximal tubules; IA, interloper artery; CV, central vein; SV, sub lobular vein; GC, germinal center 


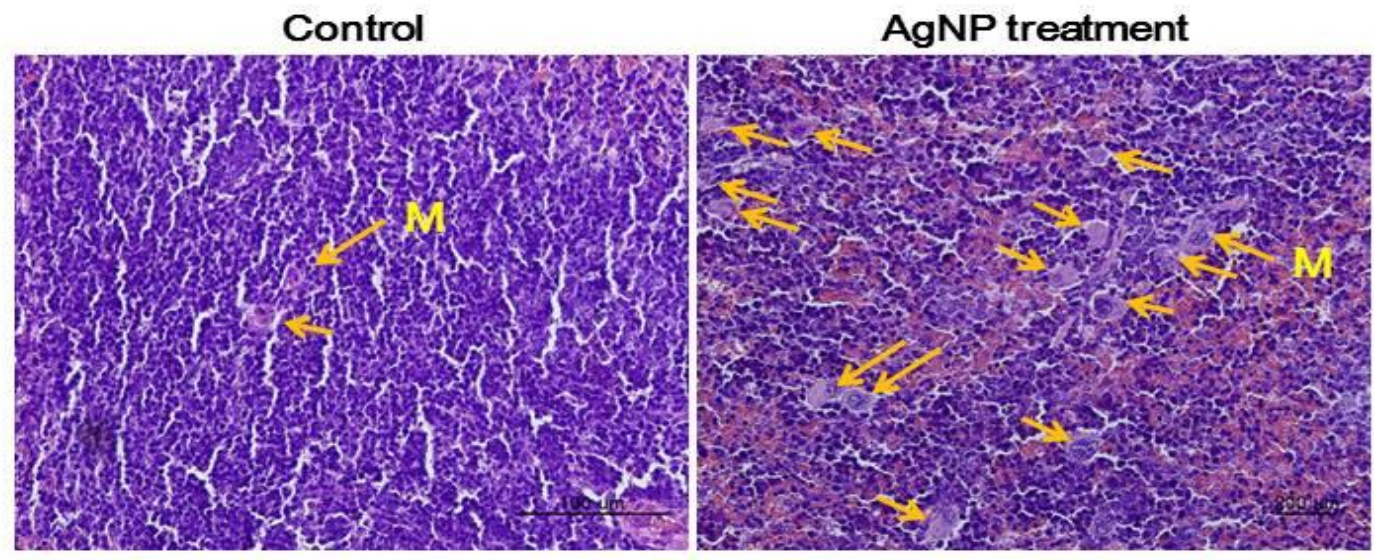

Supplementary Fig. 4. Abundance of macrophage in spleen. M indicate macrophage. 


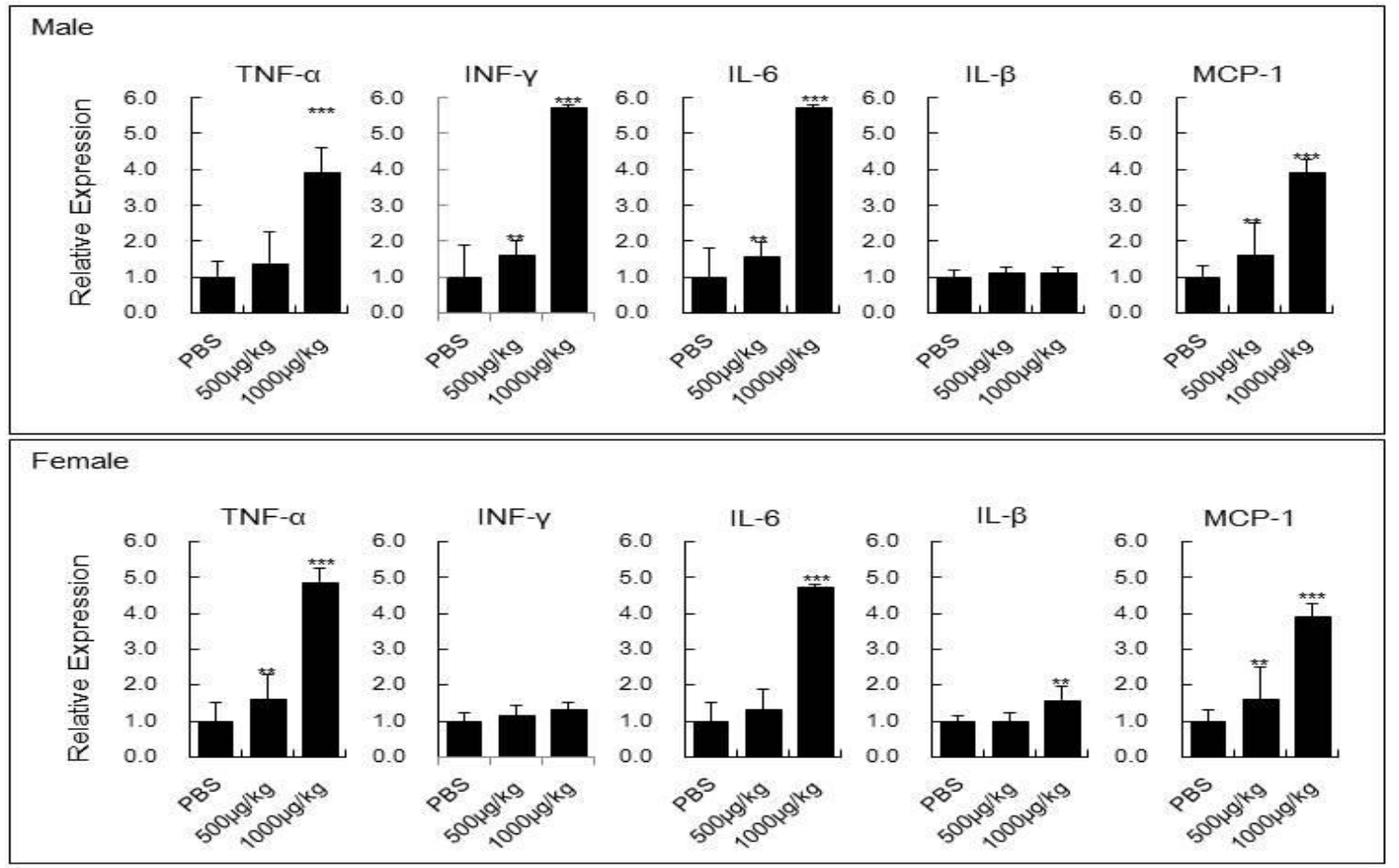

Supplementary Fig. 5. Effect of AgNPs on the expression of cytokines. Cytokine levels measured from pooled samples of 6 male (A) and female (B) mice after 15 days of treatment with $0.5 \mathrm{mg} / \mathrm{kg}$ and $1.0 \mathrm{mg} / \mathrm{kg}$ body weight of AgNPs. Experiments were performed in triplicate, and values are expressed as mean \pm SEM of three independent experiments. 
$* * P<0.01, * * * P<0.001$ compared with the control. TNF- $\alpha$, tumor necrosis factor $\alpha$; IFN- $\gamma$, interferon- $\gamma ; I L-6$, interleukin 6 ; IL- $\beta$, interleukin $\beta ; M C P-1$, monocyte chemoattractant protein 1 .
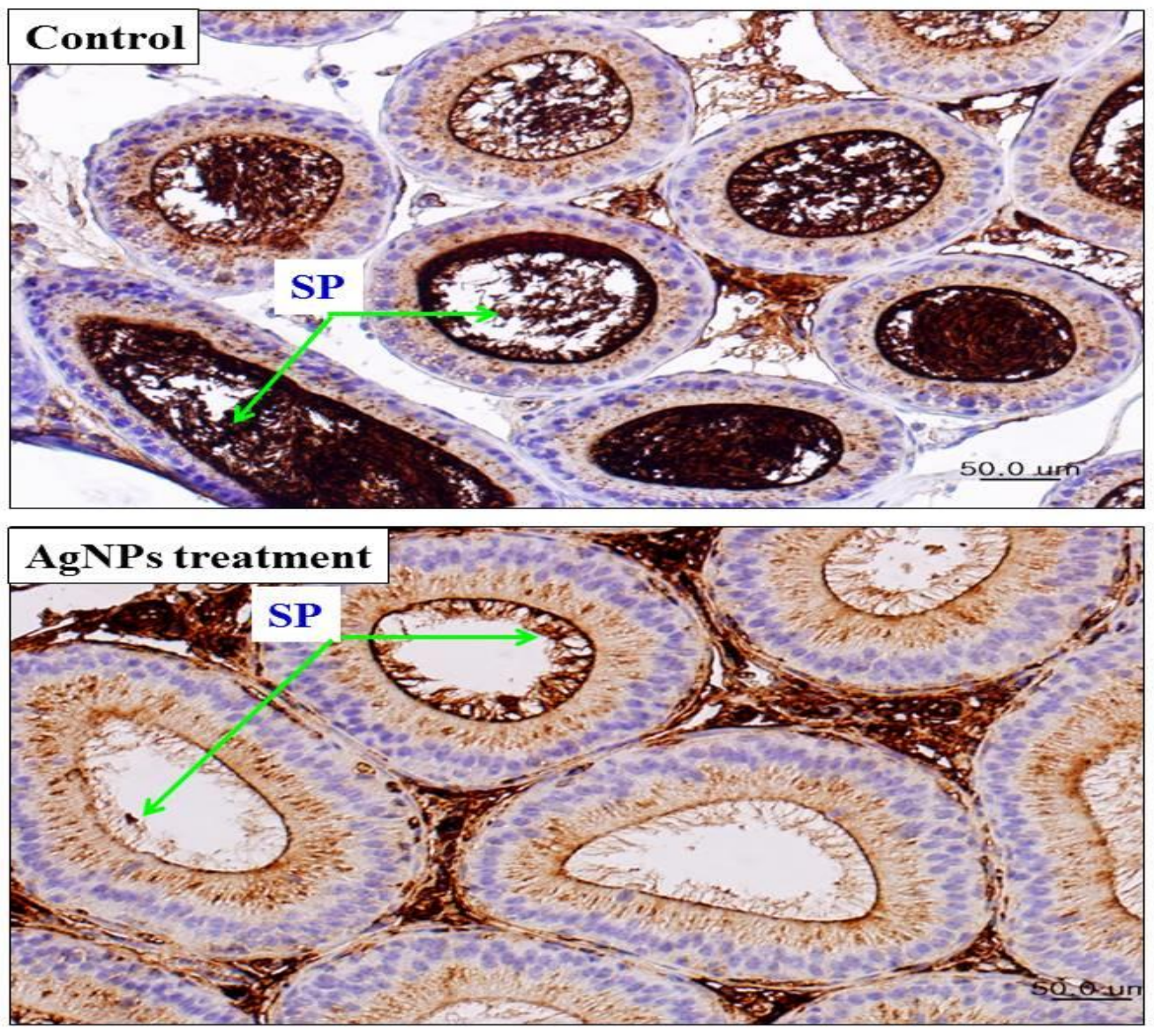

\section{Supplementary Fig. 6. Analysis of caput epididymis in control and AgNPs-treated mice.}

Dark stained signals in each tubule indicate spermatozoa. Of note, control mouse-derived tubules contained a lot of spermatozoa, whereas in AgNPs-treated shows mouse derived 
tubules have rare spermatozoa.

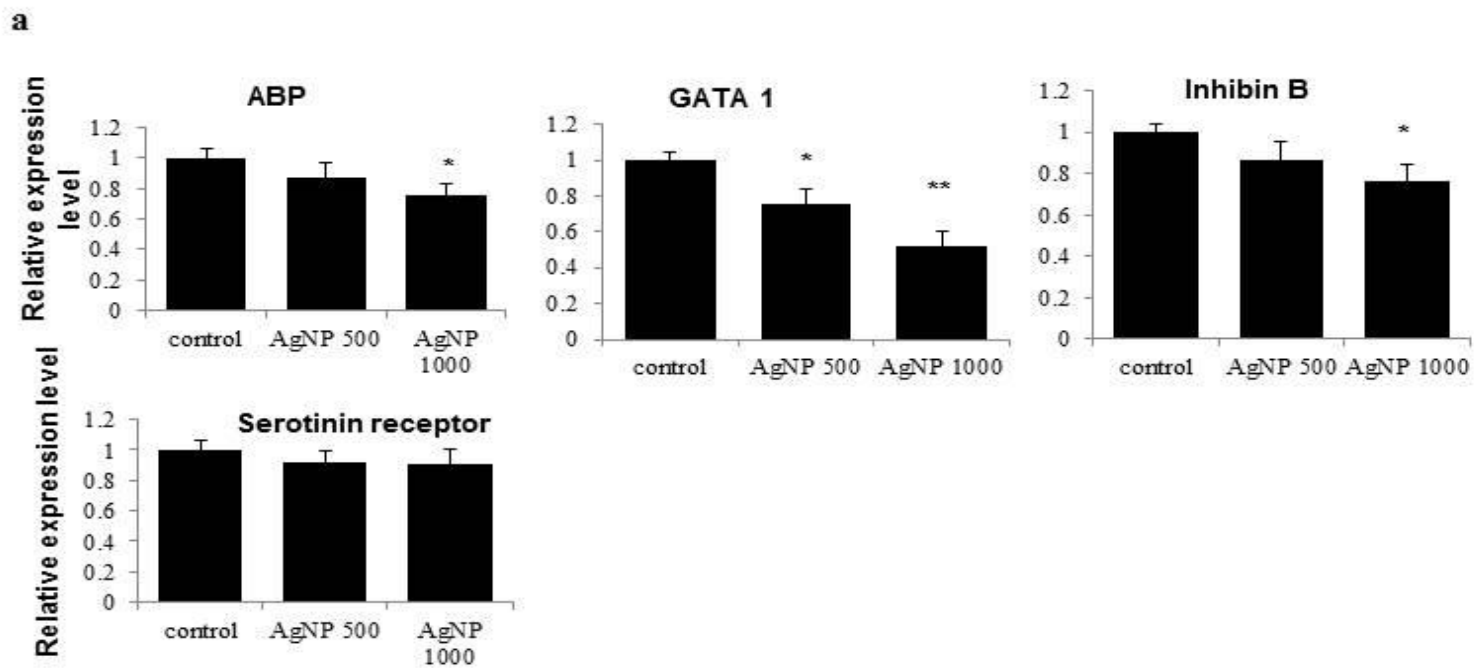

b
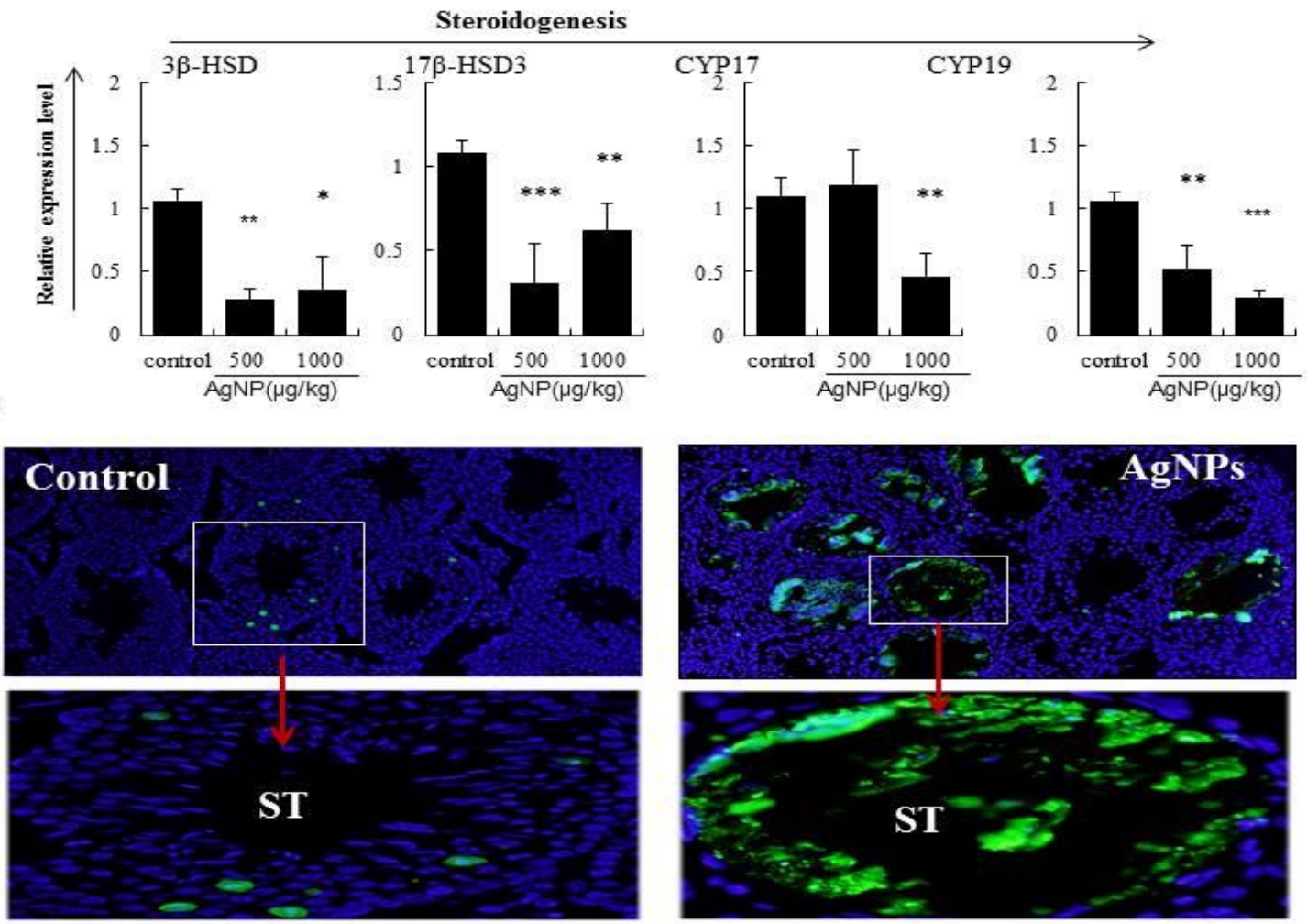
Supplementary Fig. 7. Male somatic cells (Sertoli and Leydig cells)-specific gene expressions and apoptosis analysis. (a) RT-PCR analysis of Sertoli cells-specific gene expression. Abp, Gata 1 Inhibin $b$ and Serotonin receptor. (b) Relative mRNA expression of Leydig-specific genes ( $3 \beta$-HSD, 17 $\beta$-HSD, Cyp 17al and Cyp19al) in the testes of control and AgNP-treated mice. Each target gene expression levels were normalized to GAPDH gene expression. Experiments were performed in triplicate, and values are expressed as \pm SEM of three independent experiments. $* P<0.05$, $* * P<0.01, * * * P<0.001$ compared with the control. (c) Tunnel assay analysis. Green color indicates apoptosis in male germ cells. ST, seminiferous tubule; Abp, Androgen binding protein; Gatal, GATA binding protein 1. 

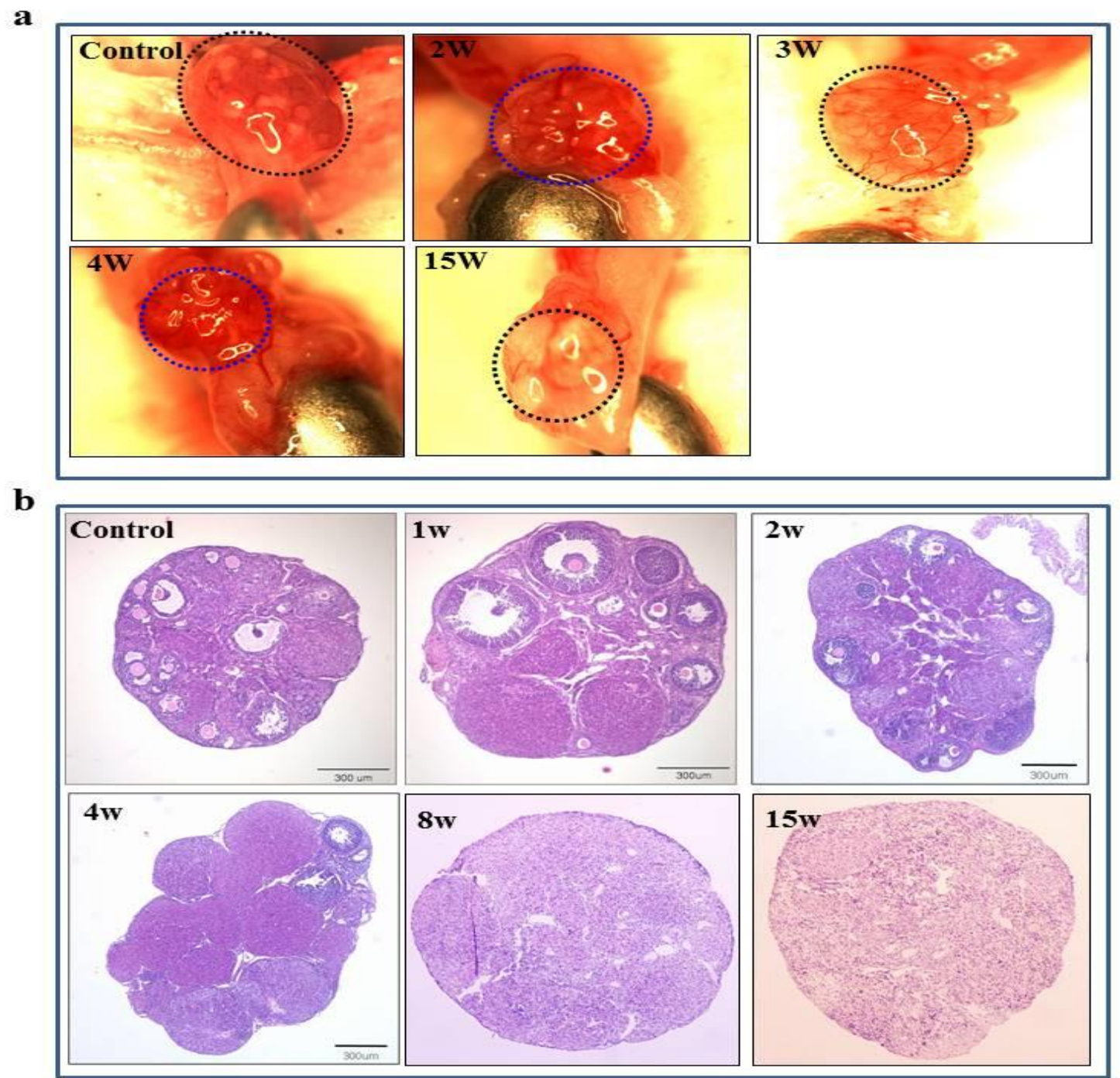

Supplementary Fig. 8. Ovary analysis after AgNPs treatment. (a) The status of ovaries condition was analyzed in control and AgNPs treated mice. Hard ovary tissues changed to soft tissues after AgNPs treatment. (b) Ovary tissue section analysis. After AgNPs treatment, the numbers of follicles in ovaries are dramatically reduced in a time-dependent manner and atrophy of developing follicles can be found in the ovaries. At 8 weeks, most of developing follicles are disappeared. 

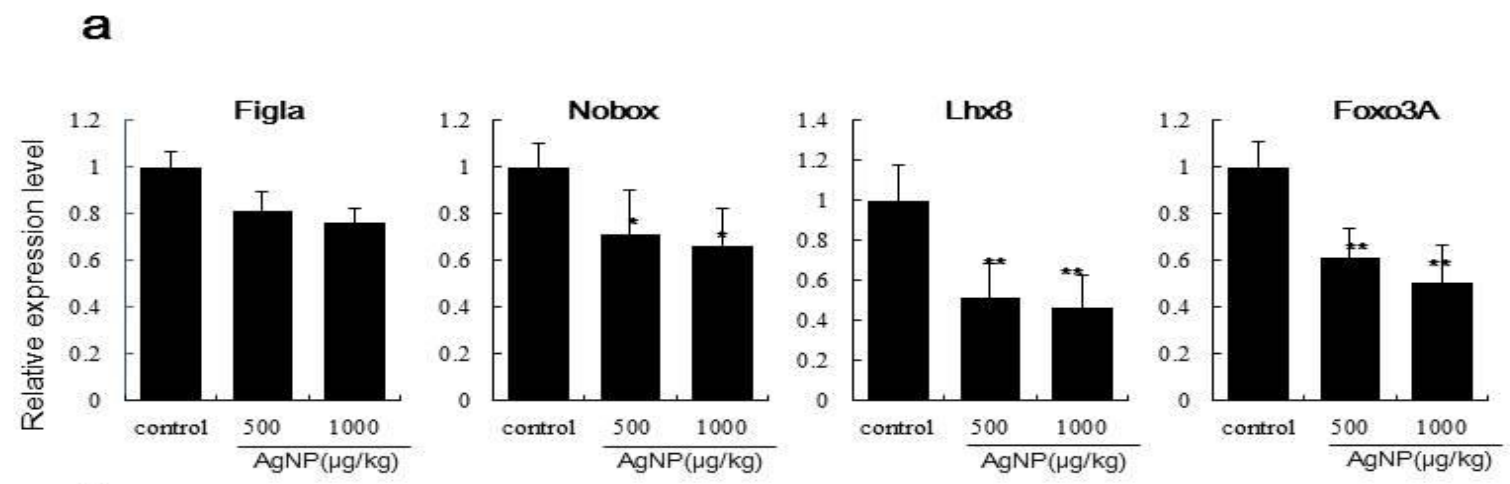

b
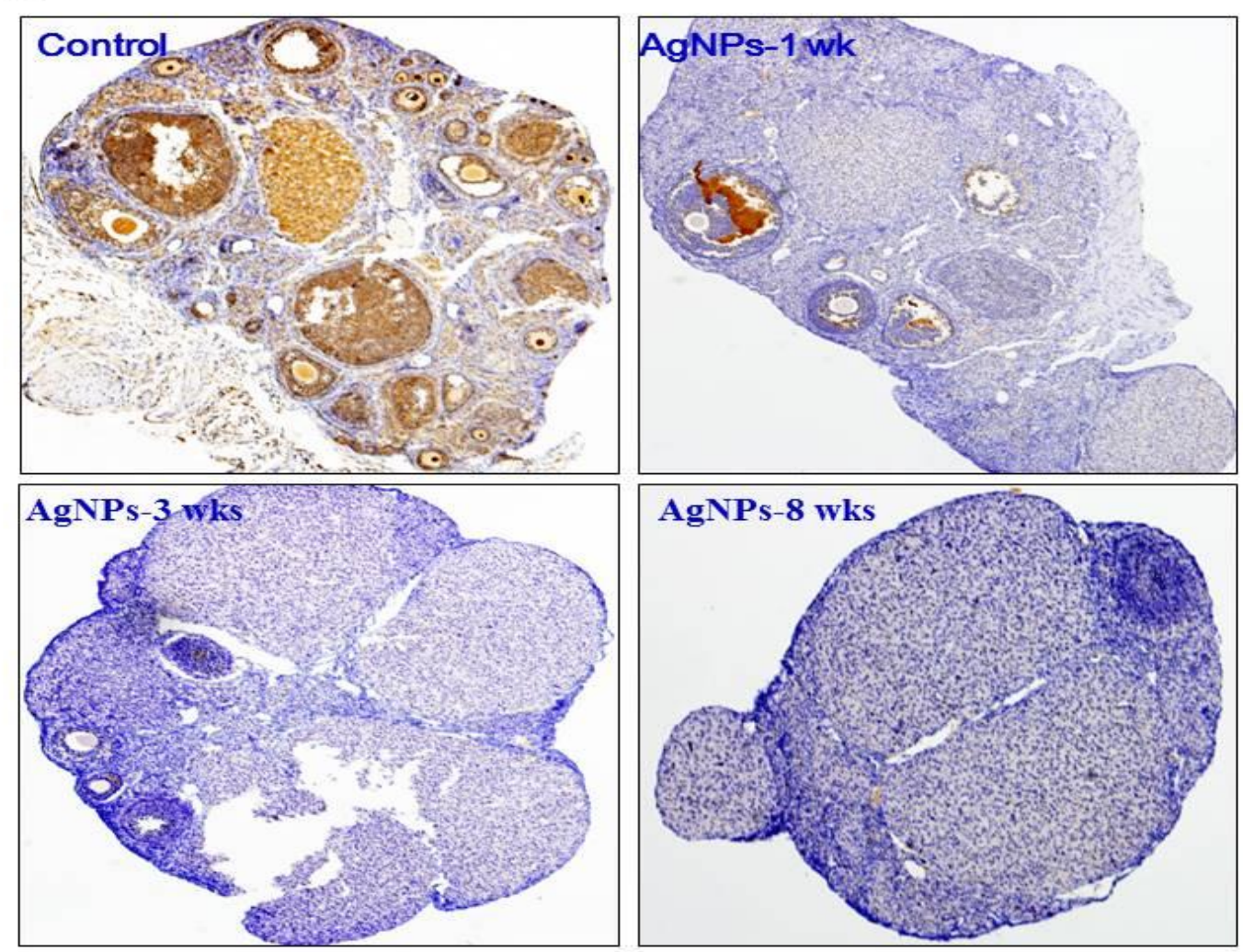

Supplementary Fig. 9. Analysis of primordial specific mRNA and protein expression.

(a) RT-qPCR analysis of female germ cell-specific genes. The expression levels of Figla, Nobox, Lhx8, and Foxo3A were significantly lower in AgNPs-treated mice than those in the control. Each target gene expression levels were normalized to GAPDH gene expression. 
Experiments were performed in triplicate, and values are expressed as \pm SEM of three independent experiments. ${ }^{*} P<0.05, * * P<0.01, * * * P<0.001$ compared with the control. (b) Lhx 8 immunohistochemistry analysis. After AgNPs treatment, the number of follicles in ovaries was dramatically reduced in a time-dependent manner. At 8 weeks after AgNPs treatment, Lhx 8 positive signals were not detected in the ovaries. Nobox, newborn ovary homeobox; Lhx8, LIM homeobox 8. 


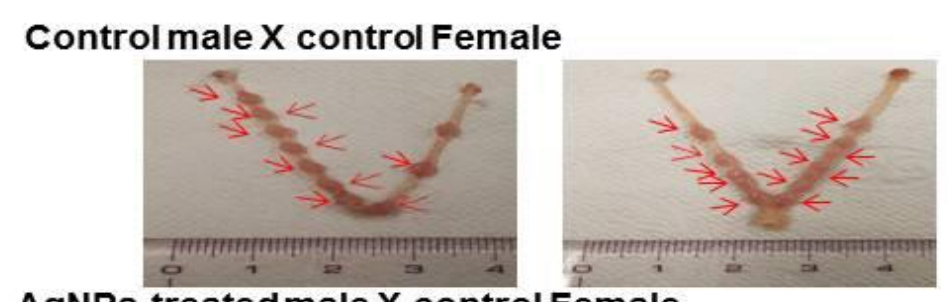

\section{AgNPs-treatedmale $X$ control Female}
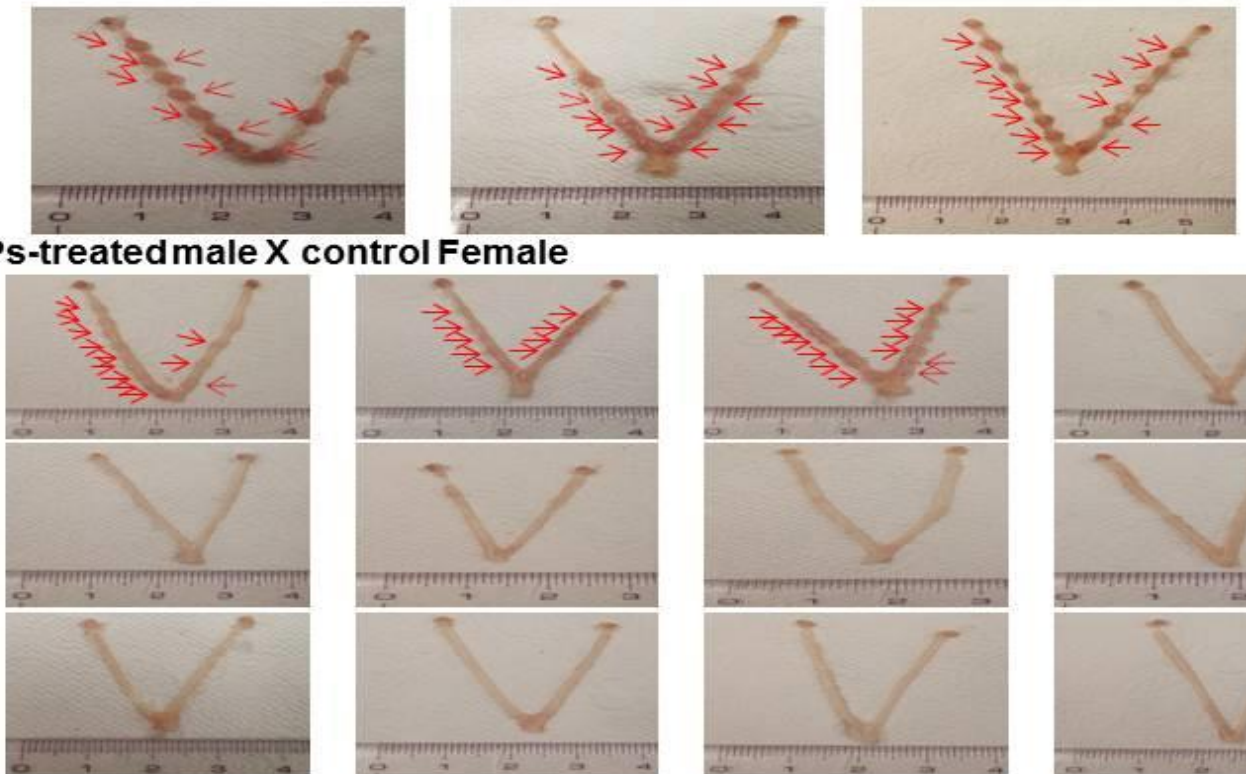

Control male X AgNPs-treated Female
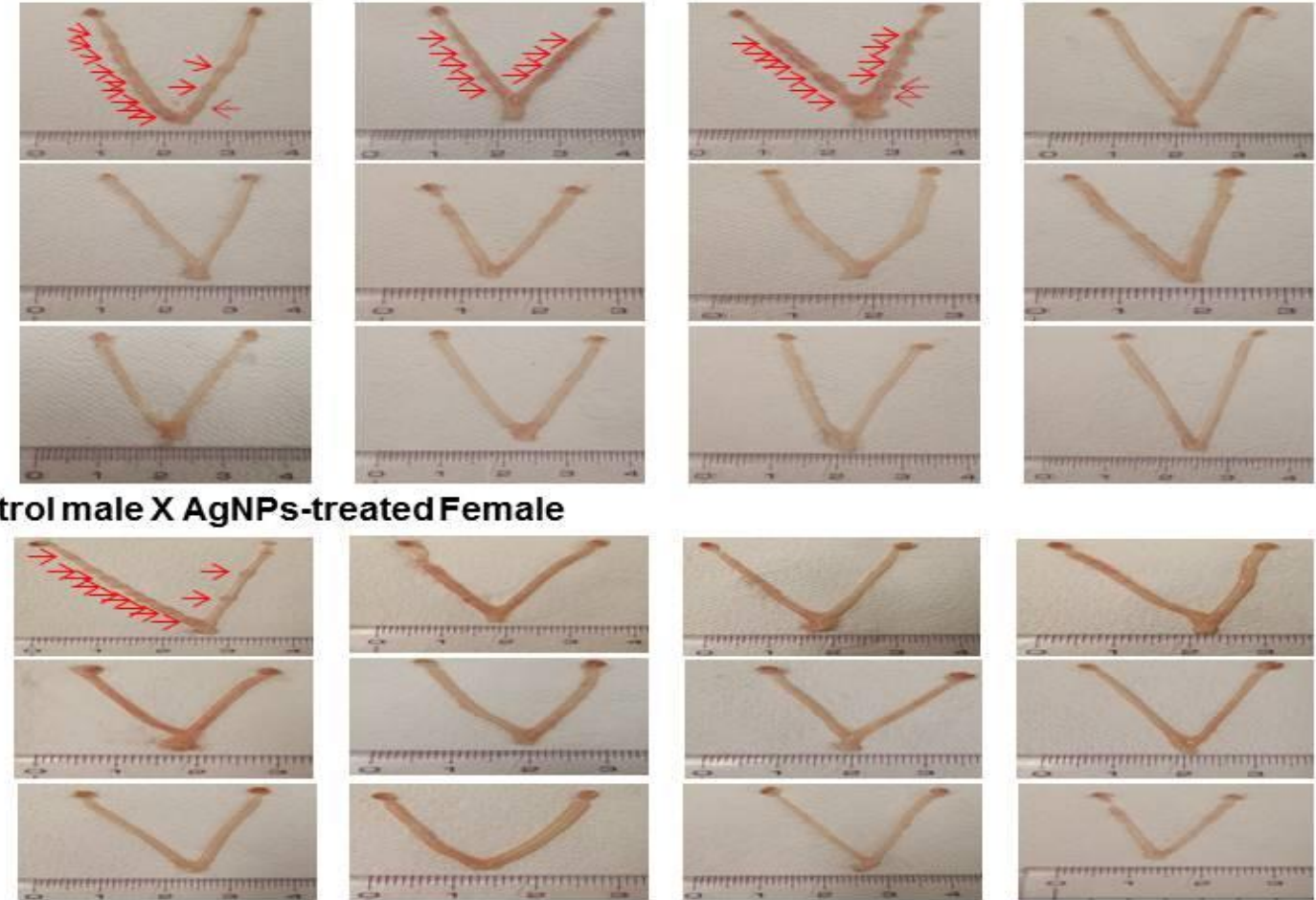

AgNPs-treatedmale X AgNPs-treated Female
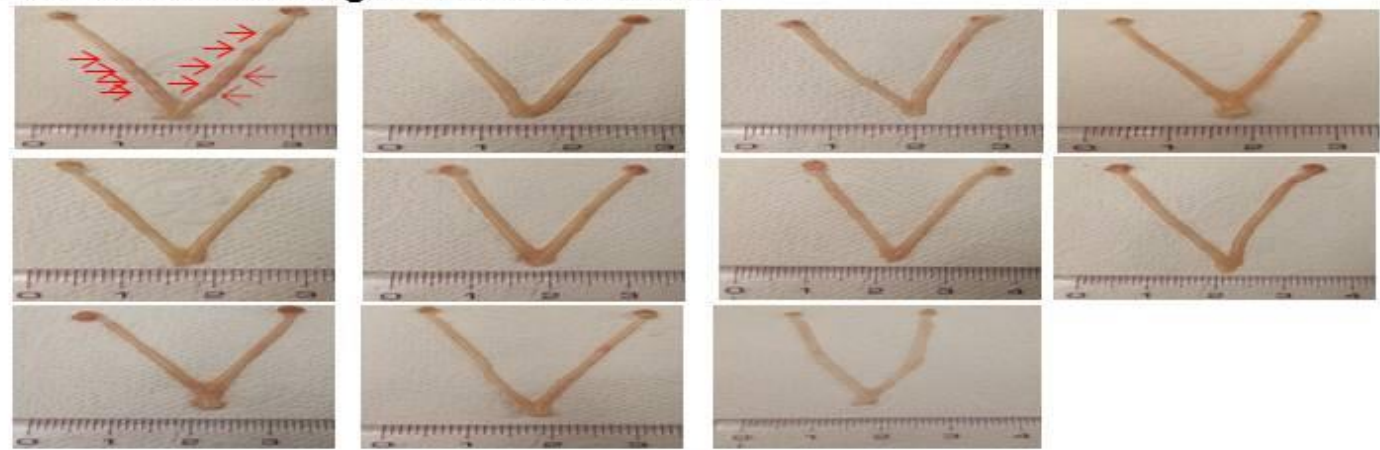

Supplementary Fig. 10. Representative images of pregnant uterus, which contains developing or arrested/aborted fetuses after mating with AgNPs-treated female or male mice. 


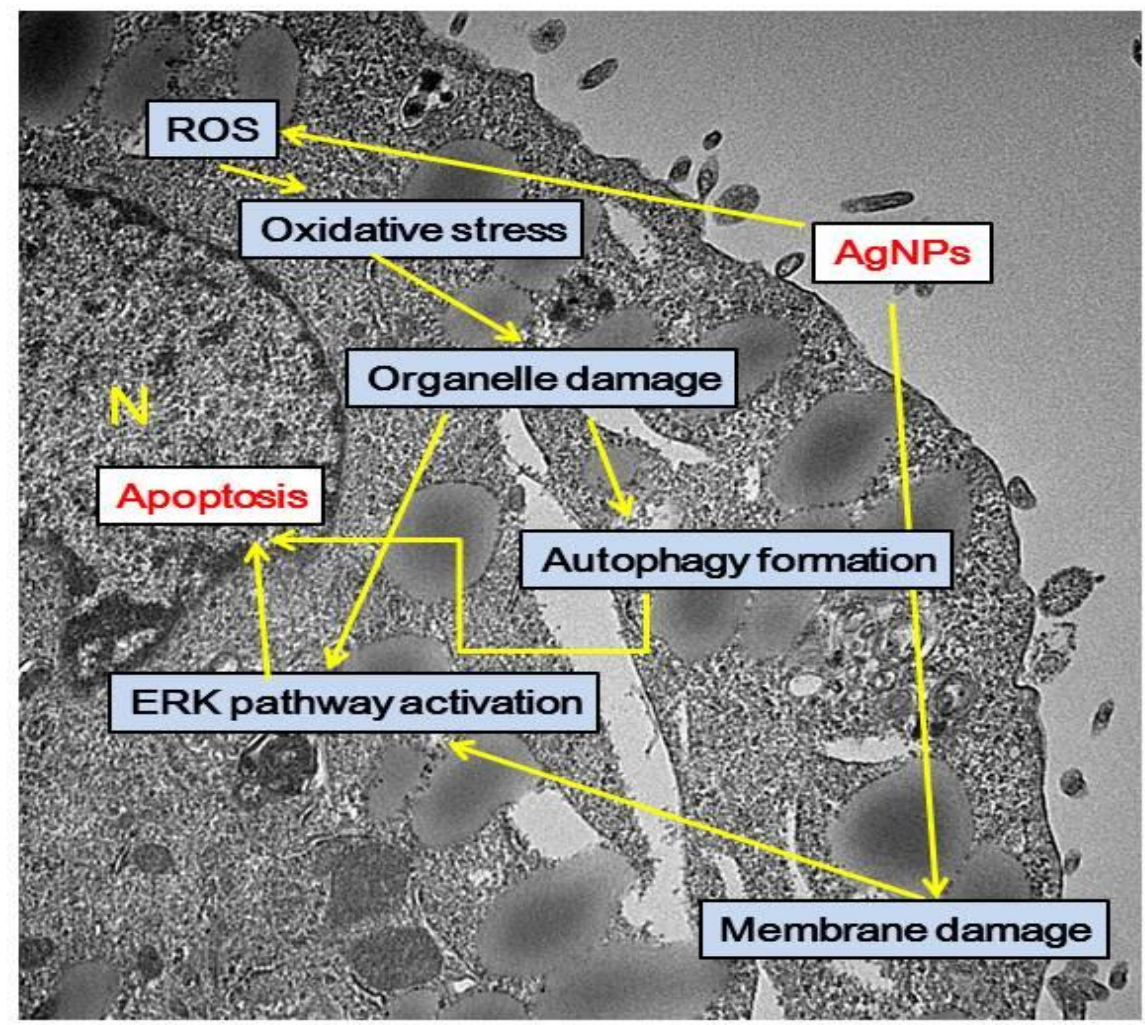

Supplementary Fig. 11. The hypothetical model to explain the mechanism of AgNPs induced cell death in TM4 cells. We propose AgNPs induced ROS mediated apoptosis in TM4 cells involve oxidative stress, organelle damage, induction of autophagy, membrane damage, down regulation of survival protein, Akt and up regulation of p53 and cytochrome c release. The thickness of the arrows indicates the importance of the biological process 
regulated by AgNPs in the cell. N, nucleus.

References

GURUNATHAN, S., KALISHWARALAL, K., VAIDYANATHAN, R., DEEPAK, V.,

PANDIAN, S. R. K., MUNIYANDI, J., HARIHARAN, N. \& EOM, S. H. 2009.

Biosynthesis, purification and characterization of silver nanoparticles using Escherichia coli. Colloids and Surfaces B-Biointerfaces, 74, 328-335.

KUO, J. (2007). Electron microscopy : methods and protocols, Totowa, New Jersey, America: Humana Press.

KWON, D. N., CHANG, B. S. \& KIM, J. H. 2014. Gene Expression and Pathway Analysis of Effects of the CMAH Deactivation on Mouse Lung, Kidney and Heart. PLoS One, 9 